Journal of Case Reports 2021;11(1):67-70

\title{
Acute Liver Failure: A Rare Manifestation of Covid-19
}

\author{
Shazia Durdana, Samia Masood \\ Department of Medicine, Jawaharlal Nehru Medical College, Aligarh Muslim University, Aligarh, Uttar Pradesh, India.
}

\section{Corresponding Author: \\ Dr Shazia Durdana \\ Email: durdana786.sd@gmail.com}

This is an Open Access article distributed under the terms of the Creative Commons Attribution License (creativecommons.org/ licenses/by/3.0).

Received

Accepted

Published

November 9, 2020

February 21, 2021

March 30, 2021

\begin{abstract}
Background: The most frequent manifestations of COVID-19 occur due to involvement of lung, the SARS-CoV-2 virus may also lead to multi-system involvement including the liver damage. Case Report: We present a patient who presented with fever, multiple episodes of vomiting followed by altered mentation with deranged liver biochemistries attributable to COVID-19 infection. During hospital stay, patient was treated for COVID-19 and subsequently showed clinical improvement as well as improvement in lab parameters. Conclusion: This case report paves the way for further studies to determine the various mechanisms of liver involvement as well as the frequency of acute liver failure in COVID-19 and to help in better understanding of this disease.
\end{abstract}

Keywords: Acute Liver Failure, COVID-19, Lung, SARS-COV-2, Vomiting.

\section{Introduction}

SARS-CoV-2, a novel coronavirus, was identified towards the end of 2019 as the cause of a cluster of pneumonia cases in Wuhan city of Hubei Province of China and subsequently engendered a global pandemic on March 11, 2020, spreading to more than 120 countries as a major threat to public health [1,2]. Coronavirus disease 2019 (COVID-19) primarily manifests as a lung infection with symptoms ranging from mild upper respiratory infection to severe pneumonia, acute respiratory distress syndrome (ARDS), and death. A significant subset of patients may even remain asymptomatic [3]. Although the most frequent manifestations of COVID-19 occur due to involvement of lung, the SARS-CoV-2 virus may also lead to multi-system involvement [4]. The second most commonly affected organ due to COVID-19, after the lung, is the liver [5,6]. Lesser degrees of liver damage evident in the form of mild elevations in aminotransferases have been reported in previous studies [7]. However, severe hepatic dysfunction resulting in acute liver failure is rare. This paper attempts to explore the available evidences on severity of liver dysfunction induced by COVID-19 and to understand the pathophysiological mechanisms that might contribute to hepatic damage in COVID-19 as patients with hepatic dysfunction are at a higher risk of progression to severe disease, thus, necessitating the importance of identifying the factors that might contribute to detrimental effects on liver and to make an attempt to halt the progression of further liver damage.

\section{Case Report}

Our patient is a 17-year-old male who had been well until 10 days before admission, when he presented to the emergency department with fever, which was intermittent and associated with chills. Two days before admission he had multiple episodes of vomiting, associated with nausea but no abdominal pain or discomfort. His parents also noticed yellow discoloration of his eyes and skin, which was followed by progressive lethargy, confusion and altered mentation. There was no history of neck stiffness, chest pain, cough, dyspnea, abdominal distention, changes in bowel habits, dysuria, or rash. He had no relevant past medical history, 
Table 1: Liver dysfunction in patients with SARS-CoV-2 infection.

\begin{tabular}{|l|l|l|l|}
\hline & $\begin{array}{l}\text { Patients with SARS- } \\
\text { CoV-2 infection }\end{array}$ & $\begin{array}{l}\text { Patients with abnormal } \\
\text { liver function }\end{array}$ & Remarks \\
\hline Guan et al. & 1099 & $\begin{array}{l}\text { AST abnormal }(22.2 \%), \\
\text { ALT abnormal }(21.3 \%)\end{array}$ & $\begin{array}{l}\text { Elevated levels of AST were observed in } 112(18.2 \%) \text { of } \\
615 \text { patients with non-severe disease and } 56(39.4 \%) \text { of } 142 \\
\text { patients with severe disease. Elevated levels of ALT were } \\
\text { observed in 120 (19.8\%) of patients with non-severe disease } \\
\text { and 38 }(28.1 \%) \text { of } 135 \text { patients with severe disease. }\end{array}$ \\
\hline Huang et al. & 41 & $15(31.0 \%)$ & $\begin{array}{l}\text { Patients with severe disease had increased incidence of } \\
\text { abnormal liver function. Elevation of AST level was observed } \\
\text { in } 8(62 \%) \text { of } 13 \text { patients in the ICU compared with } 7(25 \%) \\
\text { of the 25 patients who did not require care in the ICU. }\end{array}$ \\
\hline Shi et al. & 81 & $43(53.1 \%)$ & $\begin{array}{l}\text { Patients who had a diagnosis of COVID-19 confirmed by CT } \\
\text { scan while in the subclinical phase had significantly lower } \\
\text { incidence of AST abnormality than did patients diagnosed } \\
\text { after the onset of symptoms. }\end{array}$ \\
\hline
\end{tabular}

AST- Aspartate aminotransferase, ALT- Alanine aminotransferase.

(Adopted from Portincasa P, Krawczyk M, Machill A, Lammert F, Di Ciaula A. Hepatic consequences of COVID-19 infection. Lapping or biting? European Journal of Internal Medicine. 2020;77:18-24.)

and his family denied the use of any prescription medications, non-prescription medications, or herbal supplements. On admission, the patient was febrile with a GCS of $8 / 15$, normal sized normally reactive pupils, presence of jaundice and no evidence of nuchal rigidity. Chest was clear with bilateral equal air entry, and abdominal examination was within normal limits.

\section{Laboratory data on presentation} demonstrated markedly deranged liver functions with serum bilirubin $9.1 \mathrm{mg} / \mathrm{dL}$, aspartate aminotransferase (AST) 660 IU/L, alanine aminotransferase (ALT) $2705 \mathrm{IU} / \mathrm{L}$ (de Ritis ratio of 0.2), alkaline phosphatase (ALP) $278 \mathrm{IU} / \mathrm{L}$, prothrombin time $23.3 \mathrm{sec}$ and an INR of 2.029. The patient tested positive for COVID-19 infection performed via RT-PCR on a nasopharyngeal swab. His serology for HbsAg and $\mathrm{HCV}$ were negative, and ultrasound (USG) demonstrated no evidence of cirrhosis. The kidney function tests revealed acute kidney injury (AKI) with a serum creatinine of $3.6 \mathrm{mg} / \mathrm{dL}$ which improved through the duration of his hospital stay, settling at $0.64 \mathrm{mg} / \mathrm{dL}$ with adequate urine output. Other routine labs like leucocytes and hemoglobin were normal, and noncontrast computed tomography head and chest $\mathrm{X}$-ray also indicated no anomalies.

Repeat swabs taken 5- and 10-days postadmission also came to be positive for SARSCoV-2, during which the patient was treated with hydroxychloroquine, azithromycin and targeted therapy for hepatic encephalopathy, including lactulose. The patient showed marked improvement on day 6 of hospital admission and attained a GCS of $15 / 15$ by day 10 . He was discharged on day 15 with completely normal liver studies and a negative test for SARS-CoV-2 and followed-up with us after 14 days of home quarantine.

\section{Discussion}

COVID-19 infection usually presents with respiratory symptoms and mild liver function test abnormalities are a common accompaniment in nearly half of COVID-19 afflicted patients. However, in our case, the patient initially presented with acute non-icteric hepatitis prior 
to the development of fever and the typical respiratory manifestations of cough and difficulty in breathing. Although our patient was affected with COVID-19, however, due to the atypical presentation, our patient was evaluated for other common causes of acute hepatitis, which came out to be negative. Subsequently, his hepatitis was attributed to COVID-19 and our patient was treated with hydroxychloroquine, which has been shown to have in-vitro activity against SARS-CoV-2 [8]. Patient's symptoms improved thereafter, and in addition his liver biochemistries showed a steady improvement. It is relevant to note that our patient was not administered any antivirals during admission that could account for liver injury.

Although the main clinical manifestations of COVID-19 have been related to lung injury, which is the central cause of fatality, laboratory tests have also demonstrated significant abnormalities pertaining to other organ systems, such as cardiac and liver injuries [9,10]. Liver function abnormalities have been reported in $14 \%$ to $53 \%$ of COVID-19 patients [11]. The postulated mechanism of viral entry is through the host angiotensin-converting enzyme 2 (ACE2) receptors that are abundant in type 2 alveolar cells, as well as bile duct epithelial cells and hepatocytes [12]. Hepatic involvement in COVID-19 could be attributed to the direct cytopathic effect of the virus, sepsis, a dynamic immune dysregulation (cytokine storm) [13], or drug-induced liver injury [14]. Most of the previous data concluded that hepatic dysfunction is more common and severe in critically ill patients and those with severe disease in comparison to patients with mild to moderate illness; as shown in Table 1. Most of the studies conducted so far suggest correlation between the severity of COVID-19 infection and the occurrence of hepatic dysfunction. Interestingly, our case report depicts a patient of COVID-19 exhibiting acute liver failure with hepatic encephalopathy and coagulopathy complicated by AKI but having mild COVID-19 infection. Although, most of the prior studies have demonstrated a correlation between severity of hepatic dysfunction and severity of SARS-CoV2 infection [15], one study conducted by Yang et al, who attempted to unveil the clinical course and outcomes of critically ill patients with SARS-CoV-2 pneumonia, demonstrated that liver dysfunction occurred in $29 \%(\mathrm{n}=15)$ patients and there was no significant difference for incidences of abnormal liver function between survivors $(30 \%)$ and non-survivors (28\%) [16].

\section{Conclusion}

On reviewing the literature that is available till date, our case represents one of the rare instances where COVID-19 presented as acute liver failure without significant respiratory involvement. Thus, there is a need to be vigilant in any patient presenting with deranged liver function tests keeping in mind that it may be the initial presentation of SARS-CoV2, which will help in timely detection of infection that can prevent the development of lethal complications associated with COVID-19. This case report paves the way for further studies to determine the various mechanisms of liver involvement as well as the frequency of acute liver failure in COVID-19 and to help in better understanding this disease.

Contributors: SD: manuscript writing, and patient management; SM: manuscript editing, references and patient management; SD will act as a study guarantor. Both authors approved the final version of this manuscript and are responsible for all aspects of the study.

Funding: None; Competing interests: None stated.

\section{References}

1. CDC Covid 19 Response Team. Preliminary estimates of the prevalence of selected underlying health conditions among patients with coronavirus disease 2019-United States, February 12-March 28, 2020. Morbidity and Mortality Weekly Report. 2020;69(3):382-386.

2. Eurosurveillance Editorial Team. Note from the editors: World Health Organization declares novel coronavirus (2019-nCoV) sixth public health emergency of international concern. Eurosurveillance. 2020;25(5):200131e.

3. Albano D, Bertagna F, Bertoli M, Bosio G, Lucchini $\mathrm{S}$, Motta $\mathrm{F}$, et al. Incidental findings suggestive of 
COVID-19 in asymptomatic patients undergoing nuclear medicine procedures in a high-prevalence region. Journal of Nuclear Medicine. 2020;61(5):632-636.

4. Wang $\mathrm{D}, \mathrm{Hu} \mathrm{B}, \mathrm{Hu} \mathrm{C}$, Zhu F, Liu X, Zhang J, et al. Clinical characteristics of 138 hospitalized patients with 2019 novel coronavirus-infected pneumonia in Wuhan, China. JAMA. 2020;323(11):1061-1069.

5. Bangash MN, Patel J, Parekh D. COVID-19 and the liver: little cause for concern. Lancet Gastroenterol Hepatol. 2020;5(6):529.

6. Li J, Fan JG. Characteristics and mechanism of liver injury in 2019 coronavirus disease. Journal of Clinical and Translational Hepatology. 2020;8(1):13.

7. Jin X, Lian JS, Hu JH, Gao J, Zheng L, Zhang YM, et al. Epidemiological, clinical and virological characteristics of 74 cases of coronavirus-infected disease 2019 (COVID-19) with gastrointestinal symptoms. Gut. 2020;69(6):1002-1009.

8. Yao X, Ye F, Zhang M, Cui C, Huang B, Niu P, et al. In vitro antiviral activity and projection of optimized dosing design of hydroxychloroquine for the treatment of severe acute respiratory syndrome coronavirus 2 (SARS-CoV-2). Clinical Infectious Diseases. 2020 Mar 9:ciaa237.

9. Chen N, Zhou M, Dong X, Qu J, Gong F, Han Y. Epidemiological and clinical characteristics of 99 cases of 2019 novel coronavirus pneumonia in Wuhan, China: a descriptive study. The Lancet. 2020;395(10223):507513.

10. Zhang Y, Zheng L, Liu L, Zhao M, Xiao J, Zhao Q. Liver impairment in COVID-19 patients: A retrospective analysis of 115 cases from a single centre in Wuhan city, China. Liver International. 2020;40(9):2095-2103.

11. Guan WJ, Ni ZY, Hu Y, Liang WH, Ou CQ, He JX. Clinical characteristics of coronavirus disease 2019 in China. New England Journal of Medicine. 2020;382(18):1708-1720.

12. Zhang H, Penninger JM, Li Y, Zhong N, Slutsky AS. Angiotensin-converting enzyme 2 (ACE2) as a SARS-CoV-2 receptor: molecular mechanisms and potential therapeutic target. Intensive Care Medicine. 2020;46(4):586-590.

13. Mehta P, McAuley DF, Brown M, Sanchez E, Tattersall RS, Manson JJ, HLH Across Speciality Collaboration. COVID-19: consider cytokine storm syndromes and immunosuppression. Lancet (London, England). 2020;395(10229):1033.

14. Alqahtani SA, Schattenberg JM. Liver injury in COVID-19: The current evidence. United European Gastroenterology Journal. 2020;8(5):509-519.

15. Portincasa P, Krawczyk M, Machill A, Lammert F, Di Ciaula A. Hepatic consequences of COVID-19 infection. Lapping or biting? European Journal of Internal Medicine. 2020;77:18-24.

16. Yang X, Yu Y, Xu J, Shu H, Liu H, Wu Y, et al. Clinical course and outcomes of critically ill patients with SARS-CoV-2 pneumonia in Wuhan, China: a singlecentered, retrospective, observational study. The Lancet Respiratory Medicine. 2020;8:475-481. 